\title{
Cartographie de l'aléa mouvements de terrain par analyse statistique sous SIG
}

Service Aménagement et Risques naturels 13009 Marseille e.leroi@brgm.fr

\section{J.-L. FAVRE \\ S. REZIG}

LMSSMat UMR 8795

École centrale de Paris 92295 Châtenay-Malabry favre@mss.ecp.fr
L'aléa “glissement de terrain » est cartographié à partir de facteurs qualitatifs permanents déjà observés. On travaille à l'aide d'un système d'information géographique (SIG) sur une zone d'apprentissage où des glissements ont été recensés. Les observations étant très disparates et volumineuses, l'application de méthodes statistiques classique d'analyse de données pose des problèmes qui sont résolus grâce à des recodages et à la pondération des observations. Des critères scientifiques (pouvoir explicatif du modèle) mais également économiques (coût de réalisation de l'étude) permettent de sélectionner les facteurs explicatifs à retenir.

Mots-clés : mouvements de terrain, cartographie, SIG, analyse statistique.

\section{Statistical analysis based landslides hazard mapping using GIS}

Landslide hazard mapping is based on already observed permanent qualitative factors. Work is done under geographic information system (GIS) starting from training areas where landslides have been observed. The observations are very unbalanced and voluminous, and therefore the use of data analysis classical statistical methods rises problems which are solved through recoding and observation weighting.

Mathematical criteria (model efficiency) as well as economic criteria (cost of the study) can be mixed to select the explaining factors to be retained.

Key words : landslides, mapping. GIS, statistical analysis. 


\section{1}

\section{Introduction}

Les mouvements de terrain sont l'un des phénomènes géodynamiques les plus répandus dans le monde (Antoine, 1990). Ils présentent des mécanismes divers et complexes allant des chutes de blocs et écroulements rocheux, aux glissements de terrain et aux écoulements, en passant par le fauchage, les affaissements, les tassements avec ou sans dilatance. Au même titre que les autres risques naturels, mais de façon moins spectaculaire, leurs manifestations sont lourdes de conséquence avec plusieurs milliers de morts et plusieurs dizaines de millions de dollars de dégâts, dans le monde par an (Brabb, 1991). Aussi les décisions de prévention doivent résulter d'une analyse complète et la plus quantitative possible du risque tant pour caractériser l'aléa des sites et le réduire (méthodes actives) que pour caractériser leur vulnérabilité et la réduire (méthodes passives). Ainsi, les principaux Risques Naturels localisés font l'objet d'une tentative de formalisation par modélisation statistique (Favre et al., 1998).

La difficulté d'une telle modélisation probabiliste pour les mouvements de terrain (MVT) tient tout d'abord à sa composante « vulnérabilité ) (fonction définissant le pourcentage de dommage subit par un bien exposé à un mouvement de terrain d'intensité donnée) dont les études sont à l'état de balbutiement. Elle tient ensuite au grand nombre de facteurs causaux, qu'il s'agisse des facteurs permanents à évolution lente, jouant sur l'importance des phénomènes ou des facteurs déclenchants à évolution rapide, jouant sur l'occurrence des phénomènes. Le rôle de certains facteurs indirects, caractérisant qualitativement des états de la nature, est souvent mal défini. Enfin les mouvements de terrain sont des phénomènes variés et diffus, et les échantillons utilisés pour réaliser des statistiques ne sont pas toujours suffisants ou représentatifs (Terrier et al., 1993).

Trois approches principales ont été développées pour cartographier le risque MVT, malgré ces diffícultés : - l'approche par expertise sans règles préétablies (par exemple: Champetier de Ribes, 1987: Choubey et al. 1991) et avec règles préétablies (par exemple : Chang, 1991; Mompelat, 1994). C'est l'approche naturaliste conduisant à une appréciation qualitative. C'est l'approche la plus répandue :

- l'approche par statistiques univariées (par exemple : Malatrait et al., 1977 ; Focardi et Garzonio, 1988) et multivariées (par exemple: Neuland, 1976; Barros et al.. 1991: Leroi et al., 1993). C'est une approche naturaliste mais quantifiée grâce à l'observation des mouvements sur une zone d'apprentissage. C'est une approche en pleine expansion;

- l'approche analytique probabiliste (par exemple : Chowdhury, 1984; Cherubini et al., 1988). Elle nécessite un modèle analytique des phénomènes et la fonction de densité de probabilité conjointe des variables du modèle. Elle est très difficile à appliquer pour les versants naturels.

\section{2}

\section{Le but recherché}

On veut répondre ici à un besoin très pratique d'aménagement du territoire pour l'établissement de plans de prévention des risques (PPR). L'étude concerne les glissements de terrain (GT). On cherche une méthodologie pour établir des cartes économiques et faciles à utiliser avec les décideurs. On veut cartographier la probabilité d'avoir un glissement sur un site bien défini, si un événement déclenchant (séisme, fort épisode pluviométrique, action anthropique, etc.) venait à se produire. On analyse, sur une zone d'étude ou zone d'apprentissage, les glissements existants et leurs causes permanentes possibles.

L'intérêt de cette approche est:

- qu'elle traite des facteurs permanents qualitatifs généralement plus riches phénoménologiquement que des modèles analytiques forcément simplifiés :

- que ces facteurs sont en général cartographiés dans le monde entier contrairement aux variables quantitatives :

- que la variabilité des facteurs est prise en compte par des modalités :

- que mouvements de terrain et facteurs permanents sont observés :

- que la probabilité des événements déclenchants étudiée par ailleurs (autres risques naturels) peut être prise en compte in fine.

Il s'agit dès lors de modéliser la susceptibilité aux mouvements de terrain en se basant sur les observations de terrain, l'objectif consistant à définir les variables du modèle à partir des facteurs de causalité. Le modèle adopté est un modèle additif simple (Lebart et al., 1980): selon ce modèle additif (1), la probabilité de glissement en un point est égale à la probabilité moyenne sur toute la zone d'apprentissage à laquelle on ajoute ou retranche un terme correctif (contribution) dépendant des modalités de chaque facteur. On précise que, par facteur, la somme des termes correctifs est nulle.

$$
\begin{aligned}
& P f_{i t}=m+a(A)+b(B)+\ldots+e_{i j} \\
& \text { avec }: \mathrm{Sa}=\mathrm{S} \mathrm{b}=\ldots=0 \\
& S_{i} . . e_{i,}=0
\end{aligned}
$$

où : $\mathrm{Pf}_{\mathrm{ji}}$ : la probabilité conditionnelle de glissement, observée:

$m$ : le terme moyen, estimé :

$\mathrm{a}_{\mathrm{s}}$ : la contribution de la $\mathrm{i}^{\mathrm{e}}$ modalité du facteur $\mathrm{A}$. estimée ;

A, B, etc: : les facteurs, observés:

$E_{1}$ : le résidu, minimisé.

Chaque facteur est cartographié sous Système d'information géographique (SIG), un polygone représentant chaque modalité. Les variables continues comme le facteur " pente de versant » sont découpées en classes et les variables dichotomiques, telles que le facteur « réseau hydrographique »), en présenceabsence. Toutes les cartes sont croisées constituant ainsi des polygones dits élémentaires, homogènes, réalisation conjointe d'une modalité de chacun des facteurs. Ces polygones élémentaires sont ensuite croisés avec la carte des glissements de terrain (Fig. 1). On détermine ainsi la susceptibilité aux glissements de terrain comme le pourcentage de la surface correspondant à des glissements pour chaque polygone élémentaire. 


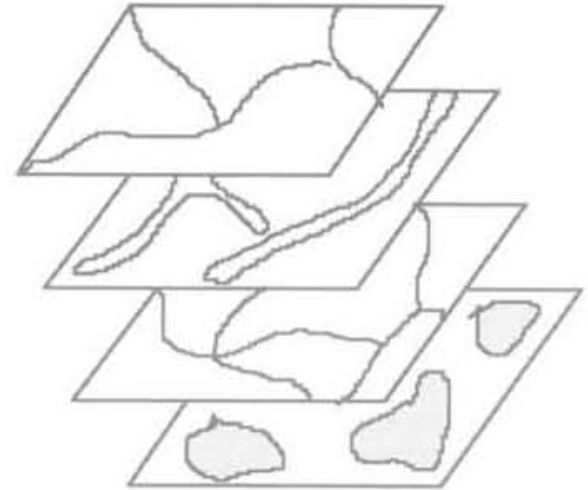

Facteur 1

Facteur 2

Facteur 3

$+$

Mouvements

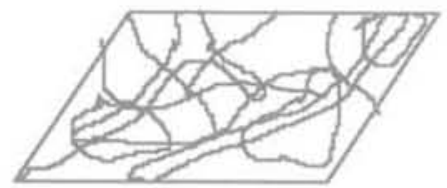

Union

Polygones élémentaires et susceptibilité. Elementary polygons and susceptibility.

On considère que ce pourcentage, rapport de la surface glissée à la surface totale du polygone élémentaire est une fréquence relative ( $\mathrm{k}$ surfaces élémentaires glissées sur $n$ surfaces possibles) qui tend vers une probabilité conditionnelle, sachant qu'un facteur déclenchant s'est produit.

Pour remédier à certains problèmes que pose cette approche, nous avons sommé tous les polygones élémentaires de même nature. On obtient ainsi une sus- ceptibilité moyenne ; on a tendance ainsi à lisser les phénomènes.

\section{3}

\section{La zone d'apprentissage et les facteurs étudiés}

\section{1}

\section{Contexte géologique}

La zone d'apprentissage est une partie (environ $30 \mathrm{~km}$ sur $15 \mathrm{~km}$ ) de la dépression du Trièves dans les Alpes françaises, déjà cartographiée par le BRGM dans le cadre du rapport Rivet (1993). C'est l'affleurement d'une épaisse série de mames sombres (terres noires) avec des alternances, vers le haut, de calcaires argileux et de marnes couronnées de bancs calcaires massifs du Jurassique supérieur. Les affleurements sont discontinus sous la couverture quaternaire pouvant atteindre par endroits $300 \mathrm{~m}$ d'épaisseur et composée :

- d'un vaste manteau d'argiles lacustres litées fréquemment surmonté de moraines :

- d'alluvions sensibles à l'érosion:

- de formations de versants.

Cette configuration est héritée de l'histoire glacière plus particulièrement du Würm. Les instabilités sont fortes ( $15 \%$ de la surface étudiée) et dues principalement aux argiles litées très sensibles à l'eau et aux terres noires du Callovo-Oxfordien, très sensibles à l'érosion.

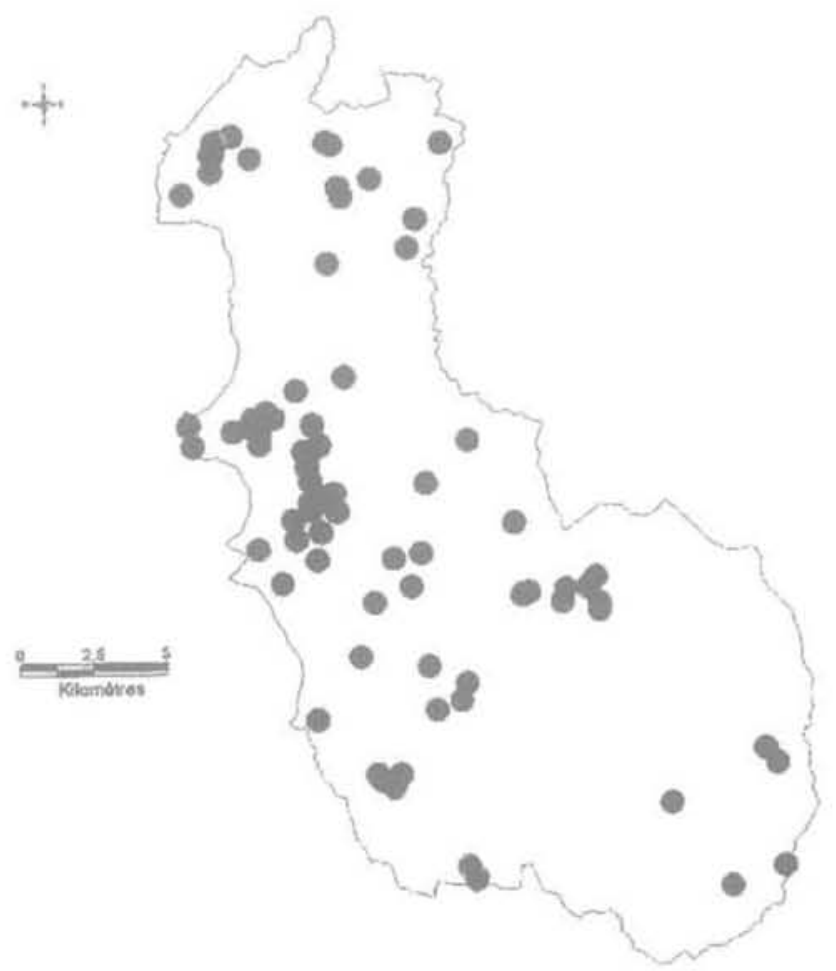

FIG. 2 Mouvements de terrain. a) fichier INVI ; b) nouvelle cartographie. Landslides maps. a) INVI data file; b) new mapping. 


\section{Les outils cartographiques}

Les différentes cartographies des facteurs étudiés ont été faites sous les SIG :

- Arcinfo, en mode « vecteurs » pour les couvertures telles que la géologie, végétation, etc. ;

- Synergis, (développé par le BRGM) en mode " raster» pour les images telles que les données des modèles numériques de terrains (MNT)

- Mapinfo, pour les sorties cartographiques.

\begin{tabular}{|c|c|}
\hline \multicolumn{2}{|r|}{$\begin{array}{l}\text { TABLEAULA Modalités du facteur " géologie ". } \\
\text { " Geologic » factor modalities. }\end{array}$} \\
\hline $\mathrm{Ag}$ & argiles litées \\
\hline C & calcaires \\
\hline $\mathrm{Cm}$ & calcaires et marnes \\
\hline e1 & éboulis vifs, actuels \\
\hline e2 & éboulis argileux, quaternaires \\
\hline $\mathrm{f1}$ & alluvions meubles \\
\hline $\mathrm{f} 2$ & alluvions hétérométriques parfois cimentées \\
\hline g & moraines glaciaires \\
\hline $\mathrm{m}$ & marnes \\
\hline s & quaternaire indiffèrencié \\
\hline
\end{tabular}

TABLEAdiB Nouvelles modalités du facteur «végétation ).

New « vegetation is factor modalities.

\begin{tabular}{l|l} 
Fa & forêts anciennes \\
Fr & forêts de recolonisation post-culturale \\
Fc & foréts recolonisation sur glissements \\
sf & pas de couvert forestier
\end{tabular}

TABLEAUIC Modalités facteur « pente du versant ». "Side slope » factor modalities.

\begin{tabular}{l|l} 
m1 & pentes faibles (de $0^{\circ}$ et $\left.10^{\circ}\right)$ \\
m2 & pentes moyennes (de $10^{\circ}$ à $\left.20^{\circ}\right)$ \\
m3 & pentes fortes (de $20^{\circ}$ à $\left.30^{\circ}\right)$ \\
m4 & pentes très fortes (de $30^{\circ}$ à $\left.40^{\circ}\right)$ \\
m5 & pentes abruptes $\left(>40^{\circ}\right)$
\end{tabular}

\section{3}

\section{La base de données et le recodage}

Les glissements de terrain : extraits de la base INVI du BRGM, ils étaient donnés par leur centre (parfois seulement le centre de la commune où ils ont eu lieu) et un rayon d'action constant de $500 \mathrm{~m}$. Une nouvelle cartographie a dû être faite au $1 / 25000$, basée sur la photo- interprétation, les observations de terrains, l'analyse des études antérieures, l'avis d'experts (Fig. 2a et b).

Les facteurs causaux : sept facteurs ont été retenus, dont deux " géologie » et " pente de versant 》 directement liés aux glissements de terrain, les cinq autres étant à tester:

1) la géologie : extraite de quatre cartes géologiques du BRGM au 1/50 000 et structurée sous Arcinfo, elle comportait cent une notations. Celles-ci ont été considérablement réduites par faciès (dix modalités cf. tableau Ia) ou par classes lithologiques (huit modalités) :

2) la végétation: extraite de la carte des peuplements forestiers de l'IFN au 1/5 000 et structurée sous Arcinfo, elle comptait douze modalités pour les premières analyses. Après discussions avec les experts du CEMAGREF, elles ont été ramenées à quatre (tableau Ib) : 3) le réseau hydrographique : c'est un réseau encaissé pouvant favoriser des glissements au niveau des berges. Seul le réseau pérenne a été retenu avec une zone d'influence de $50 \mathrm{~m}$ de part et d'autre du tracé (bufferisation). Il est extrait des fonds topographiques IGN au 1/25 000 et bufferisé sous Arcinfo avec deux modalités: pas de réseau - réseau à moins de $50 \mathrm{~m}$; 4) le réseau de failles : extrait des cartes géologiques du BRGM au $1 / 50000$ et bufferisé $(25 \mathrm{~m})$ sous Arcinfo, il compte deux modalités : pas de faille - faille à moins de $25 \mathrm{~m}$;

5) la pente de versant : extrait du MNT de l'Tsère, il a été structuré sous Synergis et compte cinq classes (tableau Ic).

Le pendage des terrains du substratum : extrait des cartes géologiques du BRGM au 1/50 000 et comportant trente-deux modalités, il a été divisé en deux facteurs dont on ignore le rôle exact :

6) I'orientation des couches à sept modalités : Est, Nord, Nord-Est, Nord-Ouest, Sud, Sud-Est, Ouest ;

7) le pendage moyen du substratum à cinq modalités comme « pente de versant $)$;

7 bis) la structure : il comporte deux modalités : structure conforme (les couches ont la même orientation que le versant) - structure non conforme (les couches n'ont pas la même orientation que le versant).

\section{4}

\section{Analyse des observations}

\section{1}

\section{Analyse des facteurs : indépendance et plan très déséquilibré}

Sur les 84000 combinaisons possibles d'une des modalités de chacun des sept facteurs, nous disposions de 3043 observations (c'est-à-dire de regroupements de polygones élémentaires de même nature), mais ayant des surfaces très différentes. Afin de mieux juger des déséquilibres éventuels, nous avons examiné pour chaque facteur la répartition surfacique de ses modalités ainsi que la susceptibilité de chacune. Ainsi, les différentes modalités de la géologie sont bien représentées (Fig. 3) mais une modalité, les argiles litées, présente un très fort pourcentage de surfaces glissées (Fig. 4). En revanche, certains facteurs sont très désé- 


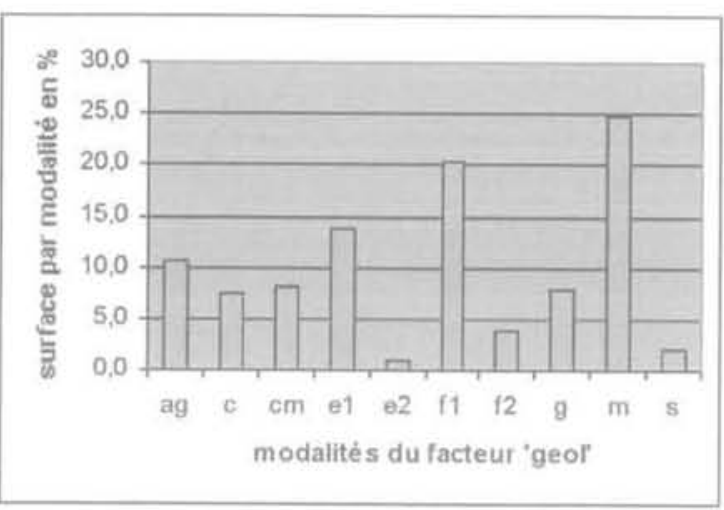

FIG.3 Répartition surfacique de la géologie par modalité.

Geology surface distribution

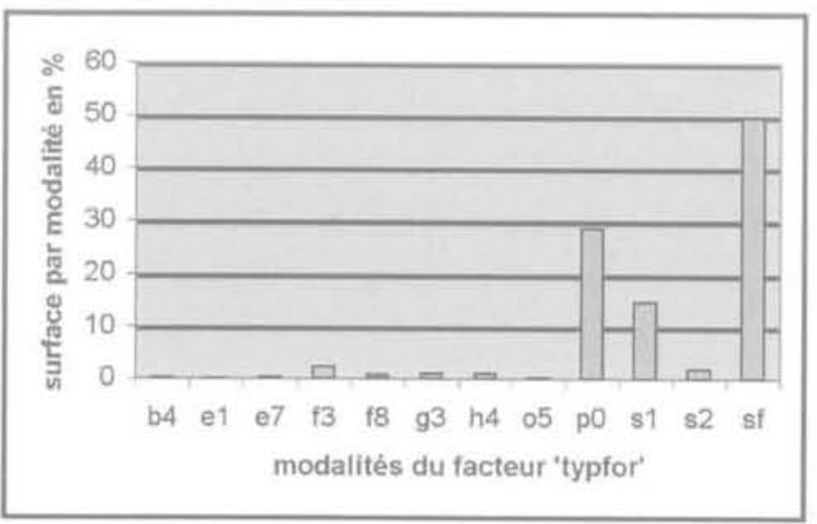

FG.5 Répartition surfacique de la végétation par modalité.

Vegetation surface distribution.

quilibrés comme la végétation (Fig. 5), suggérant des regroupements ; certaines susceptibilités sont paradoxales comme celles des pentes de versant (Fig. 6), suggérant des contextes géologiques très particuliers (argiles litées en mouvement sur des pentes de $6^{\circ}$ à $8^{\circ}$ ) ou des fortes corrélations entre facteurs jouant en sens inverse. Aussi une analyse bidimensionnelle a-t-elle été menée soit graphiquement, soit par analyse statistique du tableau de contingence (Saporta, 1990). Il en est ressorti que les différents facteurs sont a priori indépendants.

\section{2}

\section{Analyse de la susceptibilité : pondération de l'échantillon}

Il existe de grands déséquilibres dans les réalisations surfaciques des modalités de chaque facteur. Afin qu'une observation sur une petite surface n'ait pas le même poids que celle sur une grande surface, chaque susceptibilité a été pondérée. Quatre-vingt pourcent des surfaces sont inférieures à $600 \times 600 \mathrm{~m}^{2}$ soit $0,1 \%$ de la surface totale. Le plus grand polygone a une aire de $10 \mathrm{~km}^{2}$, le plus petit, de $1 \mathrm{~m}^{2}$. Les petites surfaces n'ont pas de sens physique compte tenu des échelles de cartographie. Ainsi l'adoption d'un poids pour chaque observation écarte les surfaces très petites, sans signification physique ou statistique.

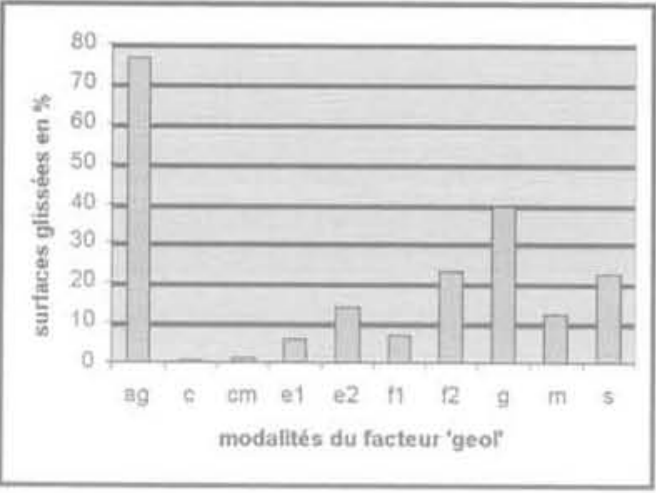

Fic.4 Susceptibilité de la géologie par modalité. Geology susceptibility.

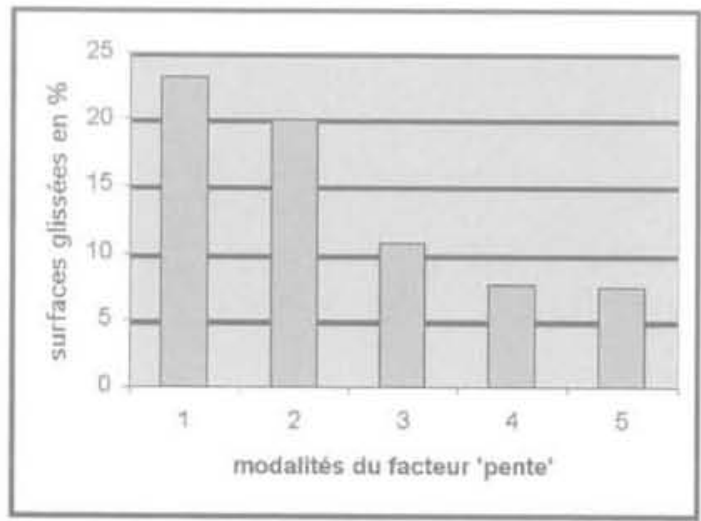

FG.6 Susceptibilité des pentes de versant par modalité.

Side slope susceptibility.

La susceptibilité a une moyenne de $17,9 \%$ avec une dispersion ou écart-type important de $26 \%$. Les susceptibilités de moins de $10 \%$ représentent $60 \%$ de l'effectif; les autres classes (par tranche de $10 \%$ ) oscillent entre 5 et $10 \%$ de l'effectif.

\section{3}

\section{Analyse conjointe de la susceptibilité et des facteurs : correspondances multiples}

Avant de proposer un modèle prédictif il faut avoir une vue globale de l'influence des facteurs sur la susceptibilité. Ceci ne peut être obtenu que par des techniques d'analyse factorielle, et ici, dans le cas de variables qualitatives, par les correspondances multiples ACM.

Le principe est de représenter les observations (ou individus) par les coordonnées qu'elles prennent sur les variables. On obtient ainsi un nuage qui, pour trois variables est un ballon de rugby aplati que l'on peut projeter dans les plans de ses trois axes principaux d'inertie, ou axes factoriels, pris deux à deux. Inversement, on peut représenter les variables par les coordonnées quelles prennent sur chaque observation. Plus deux variables sont proches, plus elles sont dépendantes l'une de l'autre, En analyse des correspondances multiples où chaque variable est un facteur éclaté suivant ses modalités, on observe la proximité des modalités que l'on chaîne. 
Fuetor 2

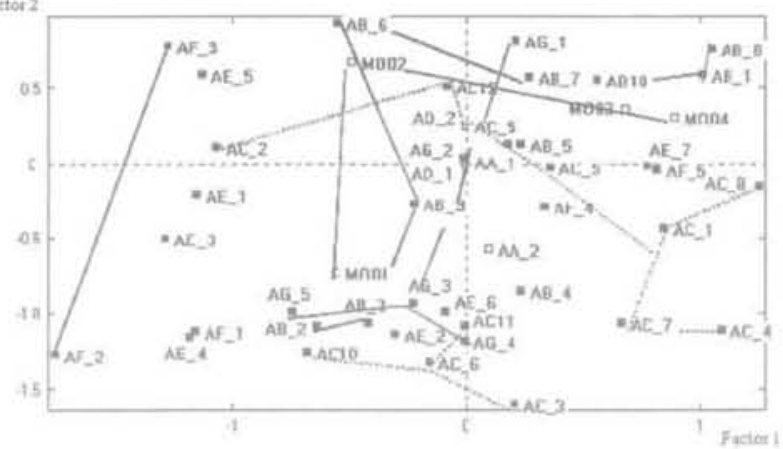

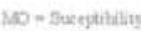
AD = Geoloig

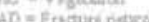

FG. 7

Analyse des Correspondances Multiples dans le plan 1-2. $M O=$ susceptibilité ; $A D=$ réseau de failles, $\mathrm{AB}=$ géologie $; \mathrm{AG}=$ pente du versant: $A C=$ végétation $: A E=$ orientation des couches; $\mathrm{AA}=$ réseau hydrographique $: \mathrm{AF}=$ pendage moyen du substratum.

$M C A$ in the 1-2 map. $M O=$ susceptibility $; A D$ $=$ fracture network: $\mathrm{AB}=$ geology; $\mathrm{AG}=$ side slope $: A C=$ vegetation $; A E=$ layers orientation: $\mathrm{AA}=$ réseau hydrographic network: $\mathrm{AF}=$ average substratum dip.

Ainsi la projection du nuage des modalités sur ses deux premiers axes factoriels (Fig. 7) montre que la géologie va bien expliquer la susceptibilité (chaînages comparables dans le plan 1-2). On voit comment d'autres facteurs la suivent aussi (la végétation) ou s'en écartent (pendage moyen du substratum). Pour d'autres, le pouvoir explicatif n'apparaît pas clairement (pente de versant), Les axes factoriels ont été construits à l'aide des sept facteurs explicatifs, la susceptibilité ayant été placée ensuite dans le graphique.

De telles analyses permettent aussi de savoir quels facteurs ont une influence significative ou pas. Ainsi, les facteurs " réseau de failles » et " pendage moyen du substratum » ont été retirés de l'étude.

\section{5}

\section{Modèles prédictifs de la susceptibilité}

Cette prévision doit être stable vis-à-vis de variations des observations en particulier lorsque le plan est très déséquilibré. D'autre part elle perd de son pouvoir discriminant (entre facteurs significatifs et facteurs non significatifs) si l'échantillonnage est de trop grande taille.

\section{1}

\section{Stabilité des analyses vis-à-vis des modalités faiblement représentées}

Le modèle à cing facteurs, UPENR, (comprenant $\mathrm{AB}, \mathrm{AC}, \mathrm{AE}, \mathrm{AG}$ et AA, cf. Fig. 7) compte 1429 observations. Le coefficient de détermination $R^{2}$, $c^{\prime}$ est-à-dire la capacité à expliquer la susceptibilité est de $82 \%$. 160 Mais certaines modalités étant très faiblement repré-
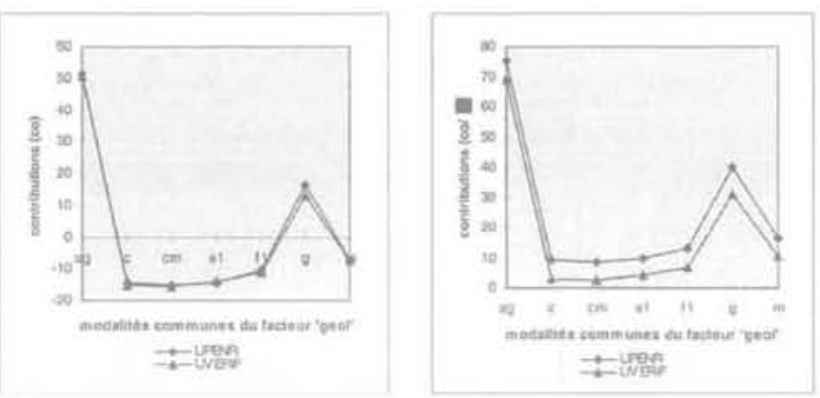

F16.8 Comparaison du modèle réduit (UVERIF) et du modèle complet (UPENR).

Comparison between the reduced model

(UVERIF) and the complete model (UPENR).

sentées, on a étudié la stabilité des analyses vis-à-vis d'elles. On a retiré toutes les modalités ne représentant que 2 à $3 \%$ de la surface totale, UVERIF. Les contributions de chaque modalité, relatives (Fig. 8a) ou absolues (Fig. 8 b), ne varient pas plus que de $4 \%$.

Ainsi, on peut considérer que le maintien dans l'analyse de modalités faiblement représentées n'a que peu d'influence. Elles seront donc conservées pour éviter un manque d'information dans l'application du modèle.

\section{2}

\section{Robustesse des analyses : poids limite et recodage}

Il s'agit de vérifier que les analyses restent stables si l'échantillonnage diminue.

Le tirage au hasard d'échantillons de plus petite taille a conné des instabilités.
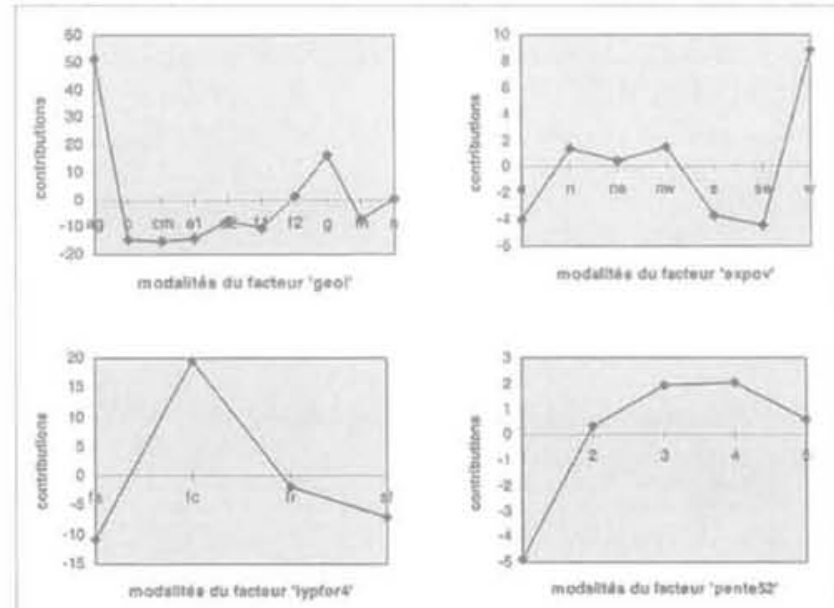

FG, 9 Contribution relative des différents facteurs ( geol $)-\alpha$ géologie $) ; ~ «$ expov $»$ $=$ " orientation des couches $n ;$; typfor $»-$ « végétation $n ;$; pente $»-\alpha$ pente du versant »), UFORA.

Different factors relative contributions (\& geol is $=\alpha$ geology $n ; \alpha$ expov $n=\alpha$ layers orientation $x$; $\alpha$ typfor $n=\alpha$ vegetation $n$; ; pente $\mathrm{y}=\alpha$ side slope n) UFORA. 
L'allègement des traitements peut être recherché dans la suppression d'individus non représentatifs et dans un recodage, d'après expertise, de certains facteurs. On s'est imposé de garder au moins deux réalisations de chaque modalité. La surface minimale retenue est de 2,4 ha. On trouve sept cent trente 730 individus. Les surfaces perdues ne totalisent que $1,34 \%$ du total. Les contributions varient peu (maximum $3 \%$ ). On est en droit de travailler sur le fichier réduit, UPELIM.

On teste avec des experts du CEMAGREF le regroupement de la végétation particulièrement déséquilibrée (on passe de douze à quatre modalités, $\mathrm{cf}$. tableau Ib) et de la pente (des pentes de $5^{\circ}$ en $5^{\circ}$ ), UFORA. Les contributions de la végétation passent 34 à $30 \%$. Les contributions de la pente suivent le sens commun. Les contributions des autres facteurs sont inchangées. Le pouvoir explicatif passe de 86 à $87 \%$. Ainsi le recodage de la végétation a un effet négligeable mais apporte un gain important en traitements. La visualisation de ces contributions est donnée par la figure 9

\section{Une cartographie pour l'aménagement du territoire}

Il s'agit ici de dégager une méthodologie pratique d'exploitation des modèles dans le cadre de l'aménagement du territoire.

\section{1}

\section{Modèle pratique optimal d'un point de vue statistique et économique}

Mais chaque facteur n'a pas le même pouvoir explicatif, c'est-à-dire le mème intérêt économique. Le pouvoír explicatif de deux facteurs n'étant pas la somme du pouvoir explicatif de chacun on a dû procéder par adjonction successive des facteurs suivant leur rang individuel. On trouve les pouvoirs explicatifs suivants : $77,8-83,6-86,1-86,5-86,8 \%$ soit un apport successif de $77,8 \%$ ( géologie $\rrbracket)-5,8 \%$ ( $\alpha$ orientation des

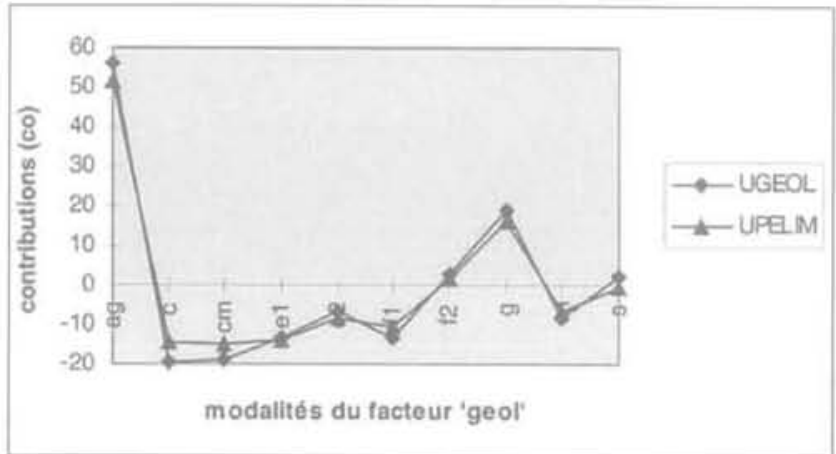

FIG. 10 Contributions comparées de la géologie (fichier UGEOL : 1088 individus - fichier UPELIM : 730 individus).

Compared geology modalities contributions (file UGEOL : 1,088 observations - file UPELIM: 730 observations).

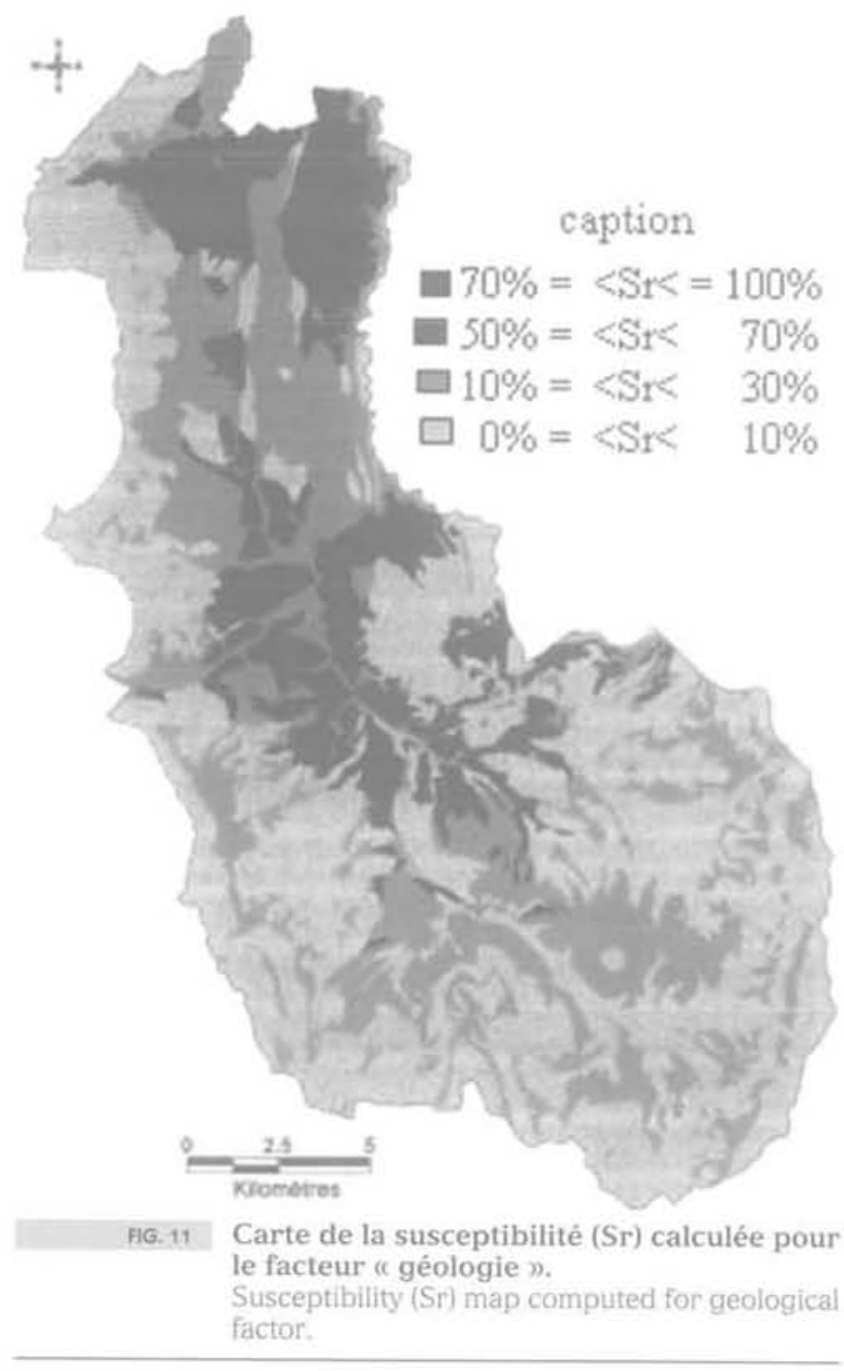

couches $»)-2,5 \%$ (« végétation $»)-0,4 \%$ ( ( réseau hydrographique $n)-0,3 \%$ ( $\alpha$ pente du versant $b)$. La géologie apparait comme très explicative, alors que la pente, tous comme les autres facteurs, l'est peu. Ceci

TABLEAUII Intervalles de confiance pour chaque modalité du facteur « géologie $»$.

Geological modalities confidence intervals.

\begin{tabular}{l|c}
$\begin{array}{l}\text { Modalité du facteur } \\
\text { " géologie n }\end{array}$ & $\begin{array}{c}\text { Intervalle de confiance } \\
\text { associé }( \pm)\end{array}$ \\
\hline $\mathrm{Ag}$ & 3,23 \\
\hline $\mathrm{C}$ & 3,81 \\
\hline $\mathrm{Cm}$ & 3,76 \\
\hline $\mathrm{E}$ & 2,8 \\
\hline $\mathrm{e} 2$ & 10,6 \\
\hline $\mathrm{f1}$ & 2,32 \\
\hline $\mathrm{f} 2$ & 5,37 \\
\hline $\mathrm{G}$ & 3,69 \\
\hline $\mathrm{M}$ & 2,09 \\
\hline $\mathrm{S}$ & 7,3 \\
\hline
\end{tabular}


vient du contexte très particulier du Trièves où on a beaucoup de glissements à faible pente, de $6^{\circ}$ à $8^{\circ}$, dans les argiles litées. Le modèle est donc cohérent avec le contexte régional.

Ainsi, pour des raisons économiques et dans ce contexte géologique particulier, le modèle retenu pour l'aménagement du territoire est le modèle à un seul facteur, la géologie, UGEOL.

\section{2}

\section{Cartographie conditionnelle des glissements du Trièves}

La géologie donne 1088 polygones élémentaires : on ne regroupe plus ceux qui sont identiques. On trouve la mème susceptibilité moyenne que précédemment, $17,9 \%$, en revanche sa dispersion a augmenté, $28,2 \%$. La première classe (de 0 à $10 \%$ ) concerne $65 \%$ de la surface totale; les pourcentages pour les neuf classes suivantes (de 10 en $10 \%$ ) chutent aussitôt en dessous de $8 \%$. Mille dix-sept observations sur les 1 088 ont une surface inférieure à $1 \mathrm{~km}^{2}$ (poids inférieur à $0,3 \%$ ). La surface maximale est de $10 \mathrm{~km}^{2}$ soit un poids de $2,8 \%$.

Le pouvoir explicatif de la géologie, passe de 77,8 à $62 \%$ ce qui paraît logique car on n'a pas lissẻ la dispersion en faisant des sommes de surfaces de même nature. Les contributions de chaque modalité sont très voisines dans les deux cas (Fig. 10). Ces contributions ont fait l'objet d'une confrontation avec trois experts de cette région (Antoine et al., 1991; Durville, 1992; Leroi et al., 1993) qui ont conforté la démarche et souligné l'intérêt du modèle comme aide à la décision. Il a été alors dressé une cartographie de la susceptibilité ou probabilité conditionnelle de glissement (Fig. 11). Les intervalles de confiance à $90 \%$ des contributions sont inférieurs à $+5 \%$ pour sept des dix modalités (tableau II).

\section{2}

\section{Guide méthodologique}

L'intérêt de développer une telle nouvelle approche pour la cartographie de l'aléa MVT réside dans les conditions de son application au vu des résultats exploitables obtenus. L'étude, qui a été réalisée dans le cadre d'une thèse (Rézig, 1998), a produit un guide méthodologique, synthétisé sous forme d'organigrammes, pour deux actions (Rézig et al., 1997):

- établir une carte de susceptibilité dans un contexte nouveau. Cet organigramme comporte dix-neuf actions dont cinq à dérivation; c'est celui de la procédure suivie :
- étendre un modèle existant à un contexte voisin. Son organigramme comporte dix actions.

\section{Conclusion}

Cette étude a permis de classer les différents facteurs, de ne conserver que les plus significatifs dans notre contexte géologique, de chiffrer les contributions de leurs modalités. Ce sont des facteurs qualitatifs, naturalistes, familiers aux experts et aux décideurs.

Elle a conduit à une carte d'aléa conditionnel. L'occurrence des événements déclenchants comme la pluviométrie ou les séismes est en général étudiée par ailleurs pour d'autres Risques Naturels. Ainsi, les résultats sont directement utilisables pour les décisions d'aménagement du territoire; néanmoins, on n'a pas distingué la nature ni les intensités des événements déclenchants qui ont provoqué les mouvements observés. Cette carte est présentée en quatre classes, ce qui la rend pratique pour les experts et les décideurs. Elle est facile à mettre à jour. Elle peut être étendue à toute zone à contexte voisin où il aurait été impossible de relever les glissements.

L'étude, menée pour les glissements peut être reproduite pour tout autre type de mouvements de terrains moyennant des zones d'apprentissage où sont cartographiés ces instabilités et un codage pertinent des facteurs causaux. L'utilisation de cartes à la même échelle suffisamment précises (1/25 000 par exemple) est recommandée.

Cette étude a montré tout le parti qu'on pouvait tirer des analyses statistiques, en particulier de l'analyse de variance, même avec des fichiers a priori énormes et des plans fractionnels très déséquilibrés. Le codage des facteurs revêt une importance d'autant plus grande que ceux-ci sont explicatifs. Néanmoins, la multiplication des facteurs, de leurs modalités, la prise en compte éventuelle d'interactions a des limites informatiques et méthodologiques, les fichiers devenant rapidement énormes. De plus, la nature nous impose des codages qui peuvent conduire à des plans très déséquilibrés, difficiles à traiter.

On a pu réduire l'échantillon en dégageant une surface minimale (rayon inférieur à $100 \mathrm{~m}$ ) à partir de laquelle les événements ne pèsent plus sur l'analyse. L'analyse décisionnelle doit être précédée d'une analyse descriptive monovariée, bivariée et multivariée. L'analyse des correspondances multiples était ici particulièrement bien adaptée.

Les analyses statistiques ont été faites avec le logiciel SPAD du CISIA-CERESTA (261, rue de Paris, 93556 Montreuil Cedex). 
Antoine P. - « Mouvements de terrain. Introduction à l'étude du phénomène s, $_{\text {. }}$ Université européenne d'été sur les risques naturels, session septembre 1990: Mouvements de terrain.

Antoine P., Monnet J., Rai N.E, Moulin C. Meriaux P, - Résultats de cinq années d'auscultation dans les argiles glaciolacustres du Triéves (Sud-Est de la France) $x$. Proceedings of the 6th International Symposium on Landislides. Christchurch (New Zealand), 1991. D. 1101-1106

Barros W.T. Amaral C. D'Orsl R.N. " Landslide susceptibility map of Rio de Janeiro y. Proceedings of the 6th International Symposium on Landslides. Christchurch (New Zealand), 1991. p. 869-871.

Brabb E.E. - a The world landslide problem 1. Episodes 14, 1991, p. 152-161.

Champetier de Ribes G. - $\alpha$ La cartographie des mouvements de terrain. Des Zermos aux PER n. Bulletin de Liaison des Laboratoires des Ponts et Chaussées 9-19, 1987, p. 150-151.

Chang S.C. - * The imprecise mapping and evaluation system for engineering geological and landslide hazard zonation $n$, Proceedings of the 6th International Symposium on Landslides. Christchurch (New Zealand), 1991, p. 905-910.

Cherubini C. Giasi C.I., Cucchiararo L. «Probabilistic analysis of slope stability in rocks v. Proceedings of the 5th International Symposium on Landslides. Lausanne, 1988, p. 361-366.

Choubey V.D.. Chandhari S., Litoria P.K. -
"Landslide hazard zonation in Uttaraka shi and Tehri districts, U.P. Himalaya, India x. Proceedings of the 6th International Symposium on Landslides. Christchurch (New Zealand), 1991, p. 911-917.

Chowdhury R.N. - « Recent developments in Jandslide studies: probabilistic methods. State of the art report 13. Proceedings of the 4th International Symposium on Landslides. Toronto, 1984 p. 209-228.

Durville J.I_ - « Mécanismes et modèles de comportement des grands mouvements de versants v. Bulletin de l'Association Internationale de Géologie de l'Ingénieur 45, 1992, p. 25-42

Favre I.L. Brugnot G. Gresillon J.M. Jappiot M. - $x$ Evaluation des risques naturels. Une approche probabiliste? Do Techniques de ITngénieur. 8-1988, C3 295 : traité Construction, 1998

Focardi P. Garzonio C A - $\alpha$ L andislide sus. ceptibility of fluvial lacustrine seciments of the upper Valdano area (Italy v). Proceedinos of the 5th International Symposium on Landslides. Lausanne, 1988, p. 135-139.

Lebart L. Morineau A., Fenelon J.P. - Traitement des données statistiques. Paris, Dunod, 1980.

Lerol E., Terrier M., Asté J.P. - « Identification des zones susceptibles d'être le siège de phénomènes d'instabilité : méthodes actuelles et perspectives D. Commission des Communautés européennes, Programme EPOCH/Projet Rivet, Rapport CCE, partie 4, vol. 2 .
1993.

Malatrait A.M., Letourneur J,, Antoine P. a Essai de cartographie systématique des mouvements de terrain dans les Alpes françaises 》. Bulletin de l'Associa. tion Internationale de Géolocie de l'Ingé. nieur 16, 1977, p. 39-46.

Mompelat P. $-\alpha$ Unités cartographiques et évaluation de l'aléa mouvements de ter. rain en Guadeloupe (Antilles francaises) 1 . Thèse de $3^{*}$ cycle de l'Université de Paris VI, 1994.

Neuland $\mathrm{H}$. - « A prediction model of landslips 11. Catena 1976, p. 215-230.

Saporta G. - Probabilités. Analyse des données et statistique. Paris. Technip, 1990.

Rezig S., Favre J. L., Lerol E, - " The probabilistic evaluation of the risk ground movement y. International Conference on Safety and Reliability. (ESREL 97). Lisbon. 1997, p. 1543-1550.

Rezig S. - $\alpha$ Modélisation probabiliste de l'alèa Mouvements de terrain. Dévelop. pement d'une méthode quantitative pour l'aide à l'expertise $\$$. Thèse de docteur de l'École centrale de Paris. 1998.

Rivet - ic Report is. Commission des com. munautés européennes, Programme EPOCH/Projet Rivet, Rapport CCE, 2 vol.. 5 parties, 1993.

Terrier M., Leroi E., Asté J.P. - u Acquisition et structuration des données pour la compréhension des phénomenes d'instabilité de versant $v$. Commission des communautés européennes, Pro. gramme EPOCH/Projet Rivet, Rapport CCE, partie 2, vol. 1. 1993 